\title{
Spherical Harmonic Theory Investigations for Spherical Antenna Arrays
}

\author{
Leonidas Marantis ${ }^{1}$, Paul Brennan ${ }^{2}$, Athanasios Kanatas ${ }^{1}$ \\ ${ }^{1}$ Department of Digital Systems, School of ICT, University of Piraeus, 80 Karaoli \& Dimitriou Street, Piraeus, Greece, \\ leomarantis@unipi.gr \\ ${ }^{2}$ Department of Electronic \& Electrical Engineering, University College London, Torrington Place, London WC1E 7JE, United \\ Kingdom
}

\begin{abstract}
Low Earth Orbit satellite communications can be significantly improved by the omni-directional beamscanning ability of the spherical antenna arrays. The signal processing of spherical antenna arrays is essentially enhanced by employing spherical harmonic theory. Spherical harmonics (or spherical modes) exploit sphere's perfect symmetry and demonstrate the feature to "reproduce" themselves in the farfield. First, this paper justifies the cost and performance superiority of the spherical arrays compared to the planar case. In addition, it presents several spherical array investigations that utilize spherical harmonic theory, offering substantial enhancement and simplifying the computational level of the array processing.
\end{abstract}

Index Terms-spherical harmonic theory, spherical modes, spherical antenna arrays, array processing, omni-directional beam-scanning.

\section{INTRODUCTION}

There are various advantageous characteristics that are associated to array antennas, such as the beam-steering feature, the multi-beam capability, the reconfigurability and the ability to develop conformal, robust and weatherproof designs [1][2]. Low Earth Orbit (LEO) satellite communications can be considered as a typical application where these features are required in order to achieve beam-scanning with full azimuth and elevation coverage. Nevertheless, planar antenna arrays experience severe radiation pattern disturbance when the pattern is scanned in wide angles [1]. Conformal arrays demonstrate additional difficulties in modelling and fabrication [3]. However, spherical antenna arrays exhibit an ideal geometry in order to generate an omnidirectional radiation pattern along with the aforementioned antenna array characteristics. Furthermore, they demonstrate high symmetry which offers the ability to expand the circular phase mode processing to the sphere and provide important computational simplifications.

This paper is organized as follows: In Section II, a basic theoretical comparison between spherical and planar antenna arrays is performed. Section III presents the adaptation of the phase mode theory concept from circular arrays to spherical arrays, by employing the spherical harmonic functions. Moreover, it demonstrates several advantages that arise from phase mode theory in specific applications. In Section IV, special emphasis is placed on the case of elements with directional radiation patterns. In Section $\mathrm{V}$, the final conclusions of our investigations are derived.

\section{SPHERICAL ANTENNA ARRAYS FOR LEO SATELLITES}

\section{A. Spherical Antenna Arrays}

When seeking to achieve a beam-scanning capability on the entire hemisphere, spherical arrays serve undoubtedly as the best available option for an Earth station LEO satellite antenna. Several other array arrangements and geometries may be able to satisfy the omni-directional requirement. However, the spherical antenna array stands out thanks to two exceptional features. The first feature is the superior efficiency of a spherical antenna array due to the fact that the directivity and gain characteristics of the array remain fixed in full azimuth and elevation coverage. This is achieved by the invariance of the array's radiation pattern when scanned in any direction. The second advantageous aspect is the reduced cost (because of the smaller number of array elements). Specifically, as demonstrated in [4], a spherical array carries approximately one fifth fewer radiators than a planar array with equivalent characteristics. Furthermore, the periodicity and the uniformity of the radiators' environment simplify remarkably the designing process and can be considered as an additional appealing characteristic of spherical arrays. Finally, spherical arrays do not demonstrate high polarization and mismatch losses in comparison to other array arrangements [3][4].

\section{B. Comparison with Planar Arrays}

This section offers a comparison of planar and spherical arrays with respect to their overall cost. This is actually achieved by comparing the required surface area of each array configuration and hence the required number of array elements. The comparison involves a spherical array with a specific active spherical region. Figure 1 illustrates the spherical array configuration of radius $R$ and its active region of $2 \alpha_{\max }$ degrees. The active area of the spherical array over the area of the planar array can be calculated by the following equation [4]:

$$
\frac{A\left(\alpha_{\max }\right)}{A_{p}}=2 \frac{1-\cos \left(\theta_{0}-\alpha_{\max }\right)}{\sin ^{2} \alpha_{\max }} \cos \theta_{0}
$$




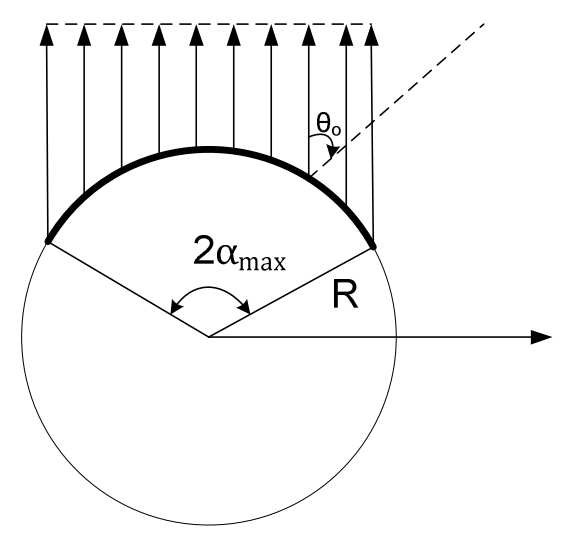

Fig. 1. Illustration of the spherical array configuration. An imaginary cone of $2 \alpha_{\max }$ degrees angle determines the active spherical region of the array.

In Figure 2, we can see the two plots (for $\alpha_{\max }=50^{\circ}$ and $\alpha_{\max }=90^{\circ}$ ) of the normalized spherical array surface area against the scan angle. As observed, for the first $75^{\circ}$ of scanning, the planar array is a less expensive solution since it occupies a smaller surface area. The spherical array is a preferable option if scanning of more than $75^{\circ}$ is required. It should be mentioned that this comparison corresponds to a theoretical investigation that considers only the scan loss $\left(\cos \theta_{0}\right)$.

\section{Spherical array normalized area versus scan angle $\theta_{0}$}

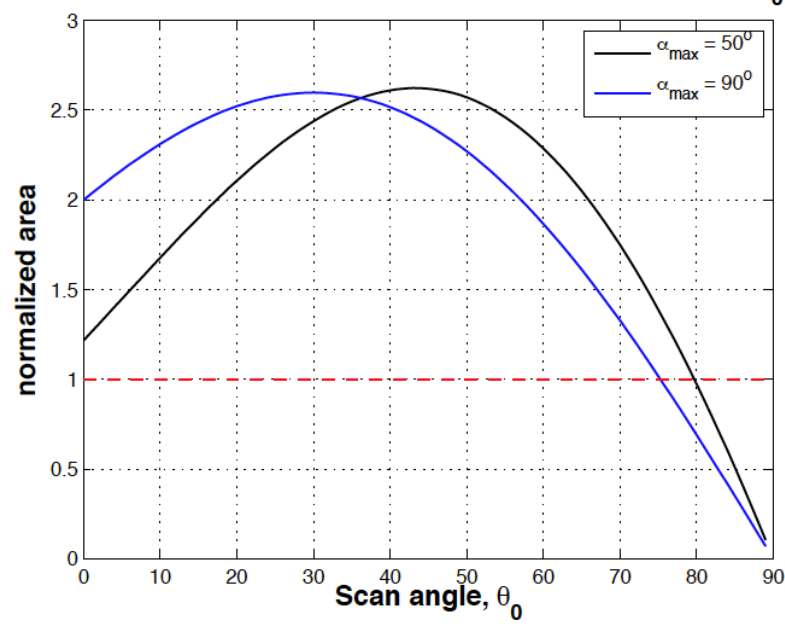

Fig. 2. Spherical array area normalized to an equivalent planar array vs. scan angle $\theta_{0}$.

In a realistic scenario though, there are a couple of additional issues that limit the performance of the planar arrays and indicate the dominance of spherical arrays, such as polarization losses and mismatch losses. Specifically, the coupling between the array elements is affected by the phase of the excitation signal and hence the scan angle. For greater than $60-70^{\circ}$ scanning angles, the directivity of the array sharply reduces resulting to a wide beamwidth and to a significant level of mutual coupling [1]. This coupling is also contributed to the impedance of each element causing a large variation of its value and a considerable mismatch effect.

\section{SPHERICAL HARMONIC THEORY}

Sir David Davies first presented the concept of phase modes in order to investigate circular arrays [5]. The Fourier analysis takes advantage of the main geometrical feature of the circle, which is symmetry (periodicity). Any function restricted to the circle can be projected in the circular harmonic basis functions (sine and cosine). Applying Fourier series that uses phase modes as its components, can decompose the excitation function of a circular array. Moreover, the phase of the far-field pattern that is associated to each phase mode is varied equivalently (in the same way) with angle, for a wide frequency range.

\section{A. Spherical Phase Modes}

Similarly to the circular Fourier transform, harmonic functions exist that exploit the symmetry properties of the sphere. These are called spherical harmonic functions (or spherical phase modes). If we consider that Helmholtz's equation has a solution in spherical polars, the spherical harmonic functions comprise the joined angular factors of this solution [6]. It can also be stated that the spherical harmonic functions demonstrate the space dependency of the scalar wave equation [7][8]. The following formula gives their mathematical definition:

$$
Y_{l}^{m}(\theta, \phi)=\sqrt{\frac{(2 l+1)(l-m) !}{4 \pi(l+m) !}} P_{l}^{m}(\cos \theta) e^{j m \phi}
$$

where $\theta$ and $\phi$ are respectively the polar and the azimuthal coordinates in the spherical coordinate system, $P_{l}^{m}$ is the associated Legendre function, $l$ is the degree and $m$ is the order of the spherical harmonic.

\section{B. Phase Mode Theory for Spherical Arrays}

The extension of the phase mode theory concept from circular arrays to spherical was first proposed by Griffiths. DeWitte, Griffiths and Brennan then proved this result and demonstrated the influence of the spherical phase modes on the far-field of the excited sphere [9]. Many of the efficient, attractive and sophisticated aspects of the array signal processing for spherical arrays derive from the concept of spherical phase mode excitation. Various and important applications are based on the feature of spherical phase modes to structure a dense basis in order to decompose any desired function into spherical harmonics, as well as on their orthogonality property. If the controls of the array are designated to the spherical phase modes, an adaptive digital algorithm can take into account the harmonic outcomes and perform several operations such as beamforming, null steering and direction finding. Furthermore, a desired farfield pattern synthesis is feasible. This is accomplished thanks to the property of each spherical harmonic of the array excitation to generate an equivalent spherical harmonic in the far-field. As a result, we can use the aforementioned Fourier analysis feature for the entire far-field array pattern, since the full pattern is also a repetitive function of angle. Thus, any desired far-field pattern can be decomposed into its harmonic terms and then it can be synthesized by separately exciting 
each of these harmonic terms and by observing ("recording") the suitable spherical Bessel factor that associate that specific harmonic's excitation to the equivalent harmonic's far-field. In addition, the complete pattern can be steered in full azimuth and elevation coverage without observing major alterations, thanks to the rotational invariance of the spherical harmonic functions.

The spherical array pattern is calculated by the summation of all the spherical harmonic terms, each of them given in the following equation [9]:

$$
D(\theta, \varphi)=j^{l^{\prime}} j_{l^{\prime}}(k R) Y_{l^{\prime}}^{m^{\prime}}(\theta, \phi)
$$

for all the degrees $l=0,1,2, \ldots, \infty$ and for all the orders $-l \leq$ $m \leq l$. As we can see in eq. (3), the spherical phase mode (and thus, the far-field pattern) is related (dependent) to a spherical Bessel function of the first kind and of order $l$ (harmonic coefficient). Since $j_{l}(k R)$ decreases sharply and acquires insignificant values for all the order values $l$ that are greater than $k R$, we can assume that, for $l>k R$, the higher order Bessel functions are negligible. A common criterion in order to truncate the Fourier series and estimate the maximum order mode $L_{m}$ that is effectively present in the spherical array excitation is $L_{m}=\lceil k R\rceil$, where $\lceil k R\rceil$ corresponds to the first integer that exceeds $k R$ [10][11]. Therefore, the number of spherical harmonics that are included in the far-field pattern is up to $\left(L_{m}+1\right)^{2}$.

In addition, McPhie [11] established a truncation constant $T$ that can be introduced in the highest order estimation according to the desired accuracy, namely:

$$
L_{m}=\lceil k R\rceil+T
$$

Figure 3 includes the truncation ratio $\left(j_{l+T}(k R) / j_{l}(k R)\right)$ plotted against the order of the spherical Bessel function, $l=$ $k R$, for several different values of the constant $T$. We clearly notice that for electrically large arrays and thus, for high values of argument $k R$, the number $T$ of additional harmonic terms that should be included in the far-field estimation is also increased in order to preserve the truncation ratio (error) to a reasonably low range (below 0.1 or $-20 \mathrm{~dB}$ ). In Figure 4, the truncation error is particularly investigated for a specific spherical array case $(f=3 \mathrm{GHz}, \lambda \approx 0.10 \mathrm{~m}$, radius $R=0.4$ $\mathrm{m})$, which corresponds to an argument of $k R=\lceil 25.16\rceil=26$ for the spherical Bessel function of the first kind for several different values of the truncation constant $T$ added to the order $l=k R$. It is evident that in order to restrict the truncation error to low values (roughly under $-20 \mathrm{~dB}$ ), we have to add at least four more harmonic coefficients in the $k R$ bound (highest order of the spherical Bessel function, $l+4$ ) in the far-field summation of the harmonic terms.

A basic assumption that is made for the majority of the spherical array antennas is that their implementation is executed using directive antenna elements on a perfectly electric conductor metal spherical surface. This is considered because the signal bandwidth of the array can be fairly enhanced by the directive array radiators. To be more precise, from Equation (3), the multiplication scaling component of the spherical phase mode (spherical Bessel function), is a function of the ratio $R / \lambda$. From the above, it is concluded that the amplitude of the far-field varies rapidly with $R / \lambda$ and the available bandwidth for use is critically limited.

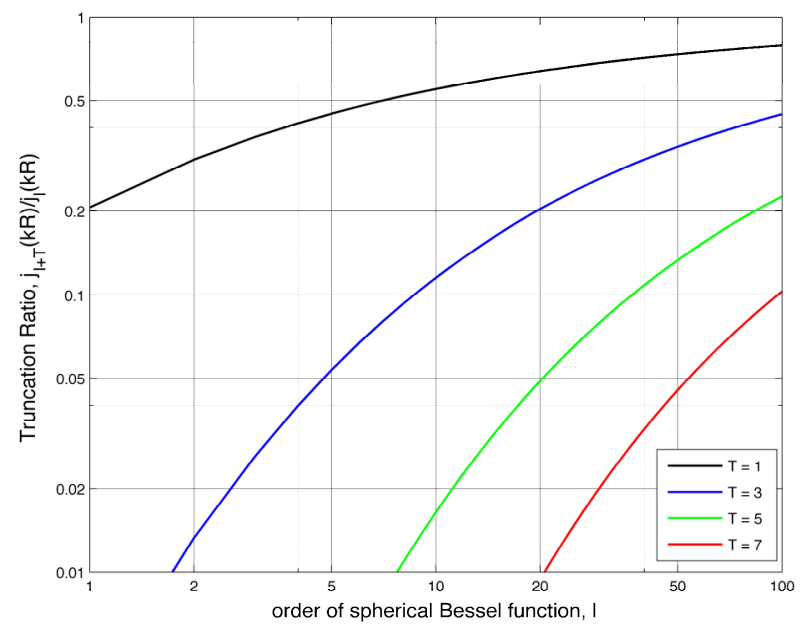

Fig. 3. Truncation error in logarithmic scale format (after normalization) is illustrated versus the order 1 of the spherical Bessel functions for all the orders that are greater than the $\mathrm{kR}$ argument.

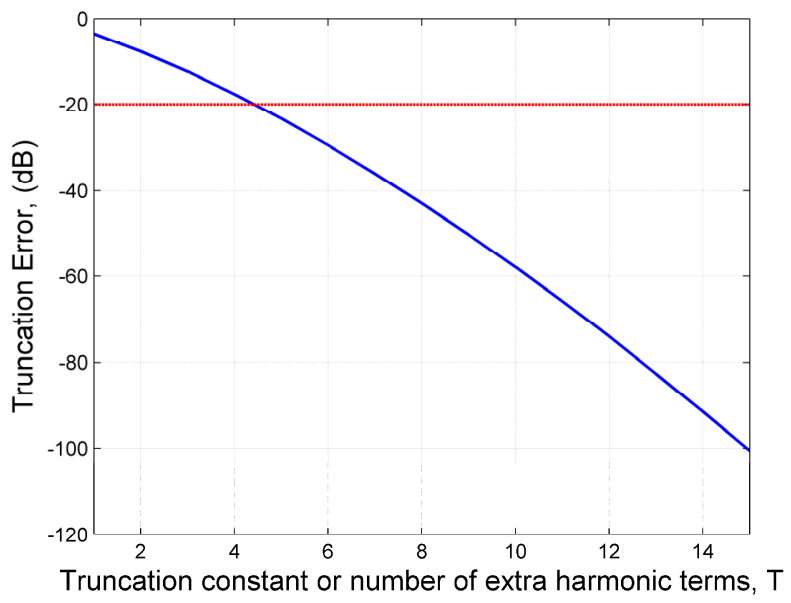

Fig. 4. Truncation error in $\mathrm{dB}$ (after normalization) is illustrated versus the number of extra modes that contribute reasonably in the far-field pattern (truncation constant), for $\mathrm{kR}=26$.

From a mathematical point of view, the aim to obtain a farfield that varies over the full sphere (full azimuth and elevation) in the same manner as the excitation function, can only be achieved if we select a ratio of $R / \lambda$ for which the same attenuation is applied to all the harmonic terms (of different degree) included in the excitation function. It is highly significant that there is no zero for any of the necessary orders $l$ in the spherical Bessel factors, for a given radius and wavelength. Indeed, this is a difficult mission to fulfill over a wide frequency band, taking into consideration the oscillatory nature of the spherical Bessel functions (see Figure 5). 


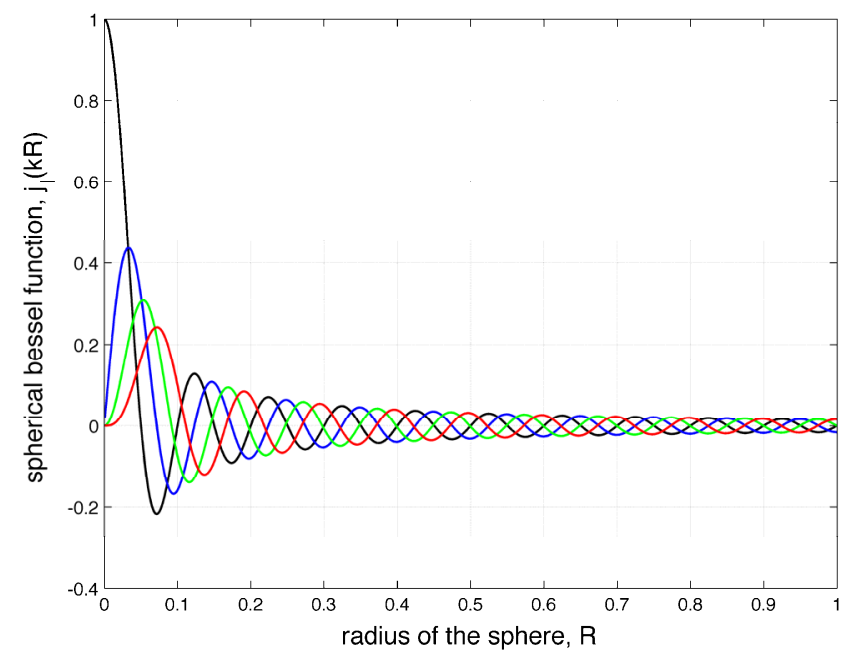

Fig. 5. The spherical Bessel functions of the first kind and of order 0 to 3 are illustrated versus the sphere radius for $\mathrm{f}=3 \mathrm{GHz}(\lambda=0.10 \mathrm{~m})$ in black, blue, red and green colors respectively.

Practically, this behavior occurs because of the serious mutual coupling effects that are observed between the isotropic radiating "elements" which are oppositely placed on the sphere, a coupling that is mainly determined by the $R / \lambda$ ratio. Specifically, since we are still investigating the continuous excitation theoretical case, we are not actually considering discrete antenna elements but the theoretical equivalent of them, which are the infinitesimal radiating segments of the sphere. Basically, the value of the $R / \lambda$ ratio that gives a zero spherical Bessel function corresponds to the case where the opposite radiating segments cancel each other. Undoubtedly, the impact on the spherical array's radiation will be great, even if the wavelength (or sphere radius) is slightly adjusted. Nevertheless, it is fortunate that this issue can and will be resolved, as follows: In the derivation, it was considered that every infinitesimal segment of the spherical surface performs like an isotropic radiator. Still, when it comes to the spherical array implementation, the mutual coupling will actually act in a less significant manner than expected. This is due to the fact that in reality directional patterns can be used, thus, an infinitesimal segment of the sphere will radiate waves that only propagate (normally to the conducting spherical surface) towards the outer space (from sphere to infinite radius).

\section{SPHERICAL HARMONICS FOR DIRECTIONAL PATTERNS}

An excellent candidate for a potential directional radiator pattern (using spherical harmonic excitations) is the raised cosine pattern that is suggested in [9]. Davies had also implied in [5][12] that a satisfactory solution to the same issue that holds for circular arrays, could be provided if we employed directional patterns. Huang proved this in theory for spherical arrays contributing the necessary mathematical estimations [13]. In this section, we implement the directional pattern case and present a few interesting simulation results of the array pattern when we vary the size and the frequency of the spherical array. Suppose that the pattern of each radiating "element" (segment) of the sphere is not omnidirectional but directional, having the shape of a pencilbeam, which practically, is a shape that reasonably approximates the patterns of many antenna elements. This pattern is derived by employing the form $(1+\cos \psi)$, that is the raised cosine pattern, pointing radially outwards from the sphere and maintaining a circular symmetry around the normal axis of the "elements". Then, we can define the farfield pattern that is stimulated by spherical harmonic $Y_{l^{\prime}}^{m^{\prime}}\left(\theta^{\prime}, \phi^{\prime}\right)$ as:

$$
D(\theta, \phi)=\frac{1}{4 \pi} \iint_{s^{\prime}}(1+\cos \psi) Y_{l^{\prime}}^{m^{\prime}}\left(\theta^{\prime}, \phi^{\prime}\right) e^{j k R \cos \psi} d s^{\prime}
$$

Subsequently, following exactly the same procedure as in [9]: (1) Bauer's plane wave expression, (2) addition theorem and (3) the orthogonality of spherical harmonics, we derive the next formula for the far-field pattern, which also includes the same harmonic that is present in the excitation function:

$$
D(\theta, \varphi)=j^{l^{\prime}}\left(j_{l^{\prime}}(k R)-j \frac{d}{d(k R)} j_{l^{\prime}}(k R)\right) Y_{l^{\prime}}^{m^{\prime}}(\theta, \phi)
$$

Equation (6) demonstrates that, when a spherical harmonic excites a directional pattern in a spherical array configuration, the far-field array mode is associated with an amplitude that does not reach zero for the entire $k R$ range of values. This is achieved because the amplitude, instead of involving one spherical Bessel function, forms a sum of them. In addition, when it comes to electrically large arrays, we can assume that $k R \gg l(l+1) / 2$ and take advantage of the spherical Bessel asymptotic forms [14]:

$$
j_{l}(x) \approx \frac{1}{x} \sin \left(x-\frac{l \pi}{2}\right)
$$

and

$$
\frac{d}{d x} j_{l}(x) \approx \frac{1}{x} \cos \left(x-\frac{l \pi}{2}\right)
$$

Hence, for spherical arrays of large dimension that operate at high frequencies, the estimation of the far-field pattern is simplified to the following formula:

$$
D(\theta, \varphi)=j^{l^{\prime}}\left(\frac{1}{k R} e^{j\left(k R-\frac{\pi}{2}\right)}\right) Y_{l^{\prime}}^{m^{\prime}}(\theta, \phi)
$$

In Figure 6, we plot the amplitude of the far-field pattern that is produced by the excitation of the $Y_{2}^{1}$ spherical harmonic against the radius of the spherical array in wavelengths $(R / \lambda)$, for three different scenarios: omnidirectional patterns, raised cosine patterns and raised cosine patterns for electrically large arrays. Firstly, we can clearly notice that, in the case of the directional pattern, the zero far- field values are eliminated, offering the array the ability of wideband patterns. This can mathematically be explained by considering that the derivative of the spherical Bessel function reaches its highest value when the spherical 
Bessel function drops to zero and the other way around, unless the unlikely case of $k R$ acquiring a value really close to zero. Thus, the factor of Equation (6), which influences the far-field pattern and consists of the spherical Bessel subtracted by its derivative, cannot give a null for reasonable size arrays of fairly high frequencies (above $2 \mathrm{GHz}$ ).

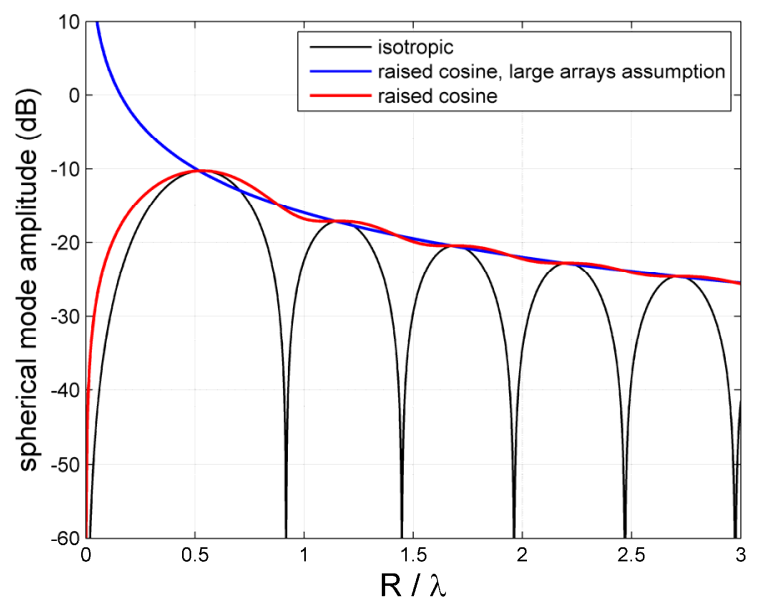

Fig. 6. The calculated far-field mode amplitude against $R / \lambda$ for the $Y_{2}{ }^{1}$ spherical harmonic excitation. The two directional patterns do not exhibit any nulls. The plot of the raised cosine pattern for large arrays is inaccurate for $\mathrm{R} / \lambda \leq 0.5$ as expected, sice the large array assumption is violated.

Secondly, according to Eq. (9), the pattern of large arrays is not affected by the degree of the spherical harmonic function that is employed and as a result, various harmonics contribute more or less equivalent amplitudes of far-field. Finally, two interesting facts can be observed in Eq. (9). The amplitude of the mode pattern declines as we increase the operating frequency of the array ( $1 / \lambda$ also increases) and the phase of the far-field harmonic is linearly proportional to frequency. As a result, the aforementioned far-field decline can be corrected by including an extra linear factor component in the array excitation that has the form of $\left(k R e^{-j k R}\right)$. This is better illustrated in Figure 7 where the two far-field amplitudes plots are plotted versus frequency for a spherical array of radius $\mathrm{R}=0.4 \mathrm{~m}$ that excites the $Y_{1}^{0}$ harmonic using a directional pattern.

\section{CONCLUSIONS}

The first part of this paper presented the comparison of planar and spherical arrays with respect to the required surface area and hence the array cost. Following, the spherical harmonic theory was introduced along with the unique properties of spherical phase modes and the attractive and efficient array processing aspects that can offer when applied to spherical array applications. Moreover, focus was placed on the criterion that is used for the selection of the maximum degree $L_{m}$ of spherical modes that have a considerable strength in the far-field of a spherical array. This study also demonstrated the requirement of using directive elements on a spherical array based on the oscillatory behaviour of the spherical Bessel functions and the bandwidth issues that this could induce.

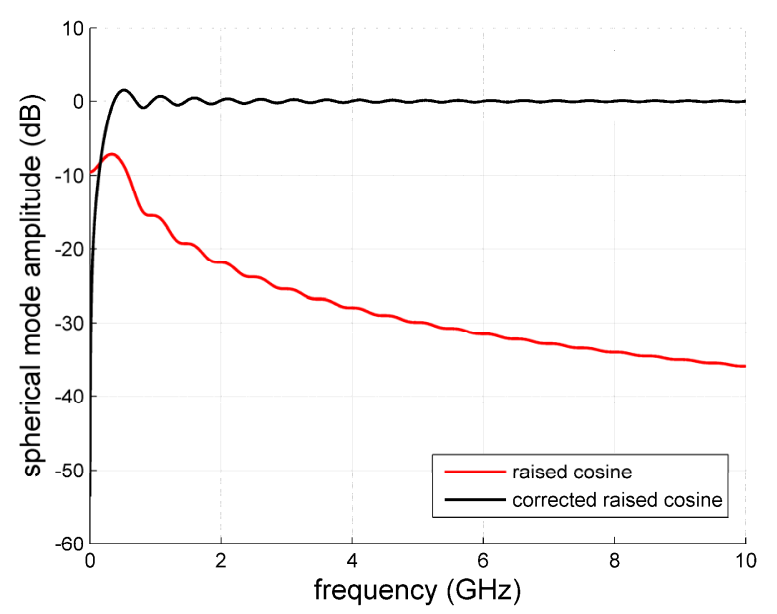

Fig. 7. The corrected far-field mode amplitude against frequency for the case of the $\mathrm{Y}_{1}{ }^{0}$ spherical harmonic excitation and $\mathrm{R}=0.4 \mathrm{~m}$, compared to the normal directional far-field pattern.

\section{REFERENCES}

[1] R. J. Mailloux, "Phased Array Antenna Handbook", Artech House Inc., Boston, 1994.

[2] R. C. Hansen, "Phased Array Antenna", John Wiley \& Sons, Inc., New York, 1998.

[3] L. Josefsson, P. Persson, "Conformal Array Antenna Theory and Design", IEEE Press Series on Electromagnetic Wave Theory, WileyInterscience, New Jersey, 2006

[4] B. Tomasic, J. Turtle and S. Liu, "The geodesic sphere phased array antenna for satellite communications and air/space surveillance - part 1", Air Force Research Laboratory (AFRL) in-house technical report, AFRL-SN-HS-TR-2004-031, January 2004.

[5] D. E. N. Davies, "The Handbook of Antenna Design - Vol. 2, chapter: Circular arrays", IEE Electromagnetic wave series, Peter Peregrinus Ltd., London, 1983.

[6] P. Young, "Helmholtz's and Laplace's equations in spherical polar coordinates: spherical harmonics and spherical Bessel functions", Physics 116C Lecture Notes, Physics Department, University of California, Santa Cruz.

[7] J. D. Jackson, "Classical Electrodynamics", John Wiley \& Sons, Inc., $3^{\text {rd }}$ edition, 1998.

[8] J. A. Stratton, "Electromagnetic Theory", International Series in Physics, McGraw-Hill Book Company Inc., 1941.

[9] E. DeWitte, H. Griffiths, and P. Brennan, "Phase mode processing for spherical antenna arrays", Electronics Letters, vol. 39, October 2003.

[10] J. E. Hansen, "Spherical Near-Field Antenna Measurements", IEE Electromagnetic Waves Series, vol. 26, Peter Peregrinus Ltd., 1988.

[11] R. H. MacPhie, "The element density of a spherical antenna array", IEEE Transactions on Antennas and Propagation, vol. 16, no. 1, pp. 125-127, January 1968.

[12] T. Rahim, D. E. N. Davies, "Effect of directional elements on the directional response of circular antenna arrays", in IEE Proceedings of Microwaves, Optics \& Antennas, vol. 129, no. 1, pp.18-22, 1982.

[13] M. D. Huang, S. Y. Tan, "An improved spherical antenna array for wideband phase mode processing", Progress in Electromagnetics Research (PIER 66), pp. 27-40, 2006.

[14] G. B. Arfken, H. J. Weber, "Mathematical Methods for Physicists", Academic Press, $5^{\text {th }}$ edition, San Diego, CA, 2001. 